\title{
Sport expertise in perception-action coupling revealed in a visuomotor tracking task
}

Maroua Mallek ${ }^{\mathrm{ac}}$, Nicolas Benguigui ${ }^{\mathrm{a}}$, Matt Dicks ${ }^{\mathrm{b},}$ Regis Thouvarecq $^{\mathrm{c}}$

${ }^{\mathrm{a} C e s a m S ~ L a b o r a t o r y, ~ C a e n ~ U n i v e r s i t y, ~ N o r m a n d y ~ U n i v e r s i t y, ~ F r a n c e ~}$

${ }^{\mathrm{b}}$ Department of Sport and Exercise Science, University of Portsmouth, United kingdom ${ }^{\mathrm{c}}$ CETAPS Laboratory, Rouen University, Normandy University, France

Corresponding author: Maroua Mallek

UFR STAPS Caen

Université de Caen Basse-Normandie,

2 Boulevard du Maréchal Juin

14032 Caen Cedex

France

E-mail: maroua.mallek@unicaen.fr

Accepted for publication in the European Journal of Sport Science on 31st August, 2017 


\section{Sport expertise in perception-action coupling revealed in a visuomotor tracking task}

\section{Abstract}

We compared the visuomotor coordination of tennis players with different levels of expertise (Super-Experts; Experts and Non-Experts) in a visuomotor tracking (VMT) task. Participants were asked to track a moving target which could rebound on the sides of a 2D screen. Results indicated that the VMT task allowed the discrimination of expertise. Multiple regression analysis revealed that performance could be explained by the temporal adaptation of participants to rebounds and the number of movement adaptations. Compared to Non-Experts, the Experts had a shorter perturbation time with higher adaptation and regulation. This corresponds to a better perception-action coupling and the predominant use of a prospective control process. Results also indicate that perception-action coupling capacities are transferable to virtual tasks, and allow us to reveal processes of visuomotor coordination that differentiate experts and novices.

Keywords: perception-action coupling; expertise; tracking task; fast-ball sports 


\section{Sport expertise in perception-action coupling revealed in a} visuomotor tracking task

Success in fast-ball sports requires highly-skilled athletes to control precisely timed visuomotor behaviors under tightly constrained spatiotemporal conditions. In baseball, for example, in order to intercept a ball thrown at $145 \mathrm{~km} / \mathrm{h}$ the spatiotemporal window for accurate performance is tightly balanced between a range of only $+/-9 \mathrm{~ms}$ and $+/-1.27 \mathrm{~cm}$ (Gray, 2002). In tennis, the returner has less than $600 \mathrm{~ms}$ to reach the ball and to return a serve delivered at $200 \mathrm{~km} / \mathrm{h}$ (Jackson \& Mogan, 2007). Thus, success in sports requires an optimal coordination between perception and action, whereby players have a very short period of time to adapt and control actions to intercept and return a projectile with a precise and powerful shot.

The study of the population of sport experts has helped to determine the processes that underpin skilled actions (Ericsson \& Smith, 1991; Yarrow et al., 2009). In ball sports, researchers have studied differences in perceptual-cognitive processes between respective experts and non-experts (Williams \& Ericsson, 2005). These efforts have contributed to a large body of literature, which indicates that experts are better than novices at anticipating the actions of opponents on the basis of contextual and biological motion information (Triolet, Benguigui, LeRunigo, \&Williams, 2013).

Expertise in ball sports can also be explained on the basis of the control and scaling of actions relative to perceptual information (Bootsma \& van Wieringen, 1990). One way to examine such relations between perception and action is to study the capacity of participants to adapt their movements relative to changes in a task with increasingly complex demands (e.g., Benguigui, Baurès \& LeRunigo, , 2008). Specific to changes in ball trajectories, many factors such as rebounds, wind, and frictions create a high level of uncertainty and require the on-line adaption of movements within a short visual-motor delay (VMD) (Benguigui, Ripoll, \& Broderick, 2003). The prospective control of movement offers a means to explain accurate 
1 behaviour in such situations as this process is based on the continuous adaptation of actions to

2 the on-going information without any need for prediction (e.g., Jacobs \& Michaels, 2006).

3 The principle is to continuously reduce or cancel the discrepancy between the actual and the necessary movement. LeRunigo et al. (2010) showed that when the velocity of a target unexpectedly changed, experts adapted their actions earlier to the new velocity. They suggested that this difference, combined with the ability to reach a high velocity of the hand after the deviation of the ball, could explain the better precision of experts in ball sports.

Given the proposed differences in the precise control of actions between experts and non-experts, the aim of the current study is to examine whether expertise in ball sport could be revealed by a visuomotor tracking (VMT) task. We expected that accuracy in the VMT task, where the goal is to pursue and to continuously match the trajectory of the target, strongly depends upon precise perception-action coupling (Le Ruingo et al., 2010). Further to studies that have examined expertise using judgments in response to simulated sport scenarios (e.g., Williams et al., 2002) or real-time interactions in sport tasks (e.g., Dicks, Button, \& Davids, 2010), we aimed to examine whether expertise can be revealed by a VMT task with strict spatiotemporal demands that require precise perception-action coupling processes.

The current study contains demanding spatiotemporal constraints during the VMT task with accelerations and sudden changes in the direction of the target following rebounds on the sides of a 2D square. As movement control for interacting with moving targets is known to be based on first-order information corresponding to the velocity of the target (e.g., Bootsma, Fayt, Zaal, \& Laurent, 1997), sudden or continuous changes in the velocity should increase the difficulty of tracking and require more adaptations and accurate regulation in movement (e.g., Le Runigo et al., 2010). Three groups of participants (Non-Experts, Experts and SuperExperts in ball sports), were tested with the aim of differentiating between Experts and NonExperts and also within Experts with different level of expertise. We expected that 
performance in the task would be a function of the level of expertise specifically in the more demanding conditions (i.e., accelerated condition and tracking following a rebound). We also expected that temporal variables such as the initiation time of the effector following the first movement of the target and the time elapsed prior to the first interception of the target would act as indicators of the inertia of perception-action. Further, movement adaptations and regulations should explain the accuracy to complete the task and the differences according to the level of expertise.

\section{Method}

\section{Participants}

Three groups were tested; the Super-Experts (Super-Exp) group included 13 international level tennis players ( 8 men and 5 women), among the best 600 in the world, $22.17 \pm 5.04$ years old; the Experts (Exp) group included 14 intermediate players (11 men and 3 women), in the range of "good” to “very good”, based on the French tennis federation's regional classification, $21.12 \pm 1.3$ years old. The Non-Experts (Non-Exp) group included 13 participants (8 men and 5 women), $23.89 \pm 5.48$ years old, who had no experience of playing interceptive sports nor did they play any sports that could be considered as "fast-ball" (e.g., tennis). Finally, as a rigorous experimental check, none of the participants reported intensively playing video games (more than one hour a day); this point was carefully considered as it is known that this could affect the findings of the VMT task (e.g., Bavelier, 2006). All the participants had normal or corrected vision. Informed consent was signed prior to testing. The study was approved by the local Ethics Committee in accordance with the Code of Ethics of the World Medical Association (Declaration of Helsinki).

\section{Experimental device for visuomotor tracking task}

We used a Samsung screen (Sync Master F2380) with an area of $50.8 \mathrm{~cm} \times 28.7 \mathrm{~cm}$ and a projection resolution of $1920 \times 1080$, a digital tablet INTUOS 4 sampled at $100 \mathrm{~Hz}$ using a stylus for tracking. Both devices were connected to a laptop, in order to project the 
1 visual scene and obtain the tracking movement data. Experimental conditions were realized

2 through custom-written software "Poursuite" developed in collaboration with Richard Kulpa and Benoit Bideau (Laboratory of Movement, Sport, Health, University of Rennes 2). The screen was positioned at a height of $93 \mathrm{~cm}$ and the screen had a dimension of $31 \times 31 \mathrm{~cm}$. This resulted in a visual angle of $23.4^{\circ} \times 23.4^{\circ}$ when the participant sat at a fixed distance of $55 \mathrm{~cm}$ from the screen.

\section{Experimental conditions}

Participants were asked to track a moving target corresponding to a red disc (radius $=$ $0.5 \mathrm{~cm}$ ) on the screen with an effector consisting of a red circle (radius $=0.6 \mathrm{~cm}$ ). The effector was controlled through the use of a stylus on the graphic tablet with direct correspondence between the stylus and the effector movement on the screen. Initially, the target could be viewed as a fixed figure in the centre of the screen appearing inside a 22.19 x $22.19 \mathrm{~cm}$ square. When the participant was ready, and after having positioned the effector on the target, the experimenter started the trial by pressing a button. The target started moving for $10 \mathrm{~s}$ after a randomized delay of between 0.5 to 2 s. After each trial, feedback was provided as a percentage value representing the duration the effector's trajectory matched that of the target during the trial.

The moving target had an initial velocity of $10 \mathrm{~cm} / \mathrm{s}$, and the velocity vector was randomly oriented in different directions on each trial. The velocity could remain constant throughout the trial (constant velocity condition) or accelerated by a constant acceleration of 5 $\mathrm{cm} / \mathrm{s}^{2}$ (accelerated condition). The acceleration vector was oriented in different directions from one trial to the next and always changed direction relative to the initial velocity vector. The moving target rebounded on the side of the square with a restitution coefficient of 1 , meaning that both velocity and acceleration were held constant. Participants performed four trials as part of a task familiarisation procedure that included horizontal, vertical and diagonal displacement trajectories of the target. Participants then completed 12 trials, six in each 
acceleration condition, which were presented in a randomized order.

\section{Dependent variables}

Five different dependent variables were recorded in order to quantify the initial temporal and kinematic characteristics of the effector: (i) Initiation time, which was the time elapsed between the first time that the target moved and the first movement of the effector; (ii) First interception time, which was the time elapsed between the first time that the target moved and the moment when the effector was equivalent to the position of the moving target for the first time; (iii) Movement time was the time elapsed between initiation time and first interception time; (iv) Peak velocity corresponded to the peak velocity of the effector that occurred after initiation time; and (v) Time-to-peak-velocity corresponded to the time of the first peak velocity of the effector after initiation time.

In a pre-analysis of the data we observed that participants were not able to track the target around the rebounds with the same accuracy as in the rest of the trials. Consequently, we divided each trial into two interlaced periods of tracking: rebound tracking (following a rebound) and free tracking (without any rebounding) (see Figure 1). To determine these periods, two intermediate variables were calculated: time of divergence and time of convergence. In order to calculate these variables, indicators angle $\alpha$ and threshold angle $\alpha$ were used, as follows: Angle $\alpha$ was the angle between the direction of the moving target and the direction of the effector at each instant. Threshold angle $\alpha$ corresponded to the angle for which we considered that the accuracy of the pursuit was significantly affected. This threshold was calculated in a free tracking area defined by a square in the centre of the square (equal to $50 \%$ of the total area of the square), where the participant's control of movement was not directly influenced by rebounds on the side of the display. The calculation corresponded to the mean angle plus two standard deviations and yielded a $25^{\circ}$ angle as a threshold for detecting a change in the accuracy of the tracking.

Time of divergence corresponded to the time when three successive angle $\alpha$ started 
1 expanding at a rate exceeding the threshold angle during the rebound time interval from -500

$2 \mathrm{~ms}$, to $+500 \mathrm{~ms}$ around the rebound. Time of convergence corresponded to the time when

3 three successive angle $\alpha$ were lower than the threshold angle, in the time interval from the

4 time of divergence to $500 \mathrm{~ms}$, after the rebound. The rebound perturbation period was

5 delimited from the time of divergence to time of convergence. The free tracking period was

6 delimited from time of convergence to time of divergence of the next rebound (Figure 1).

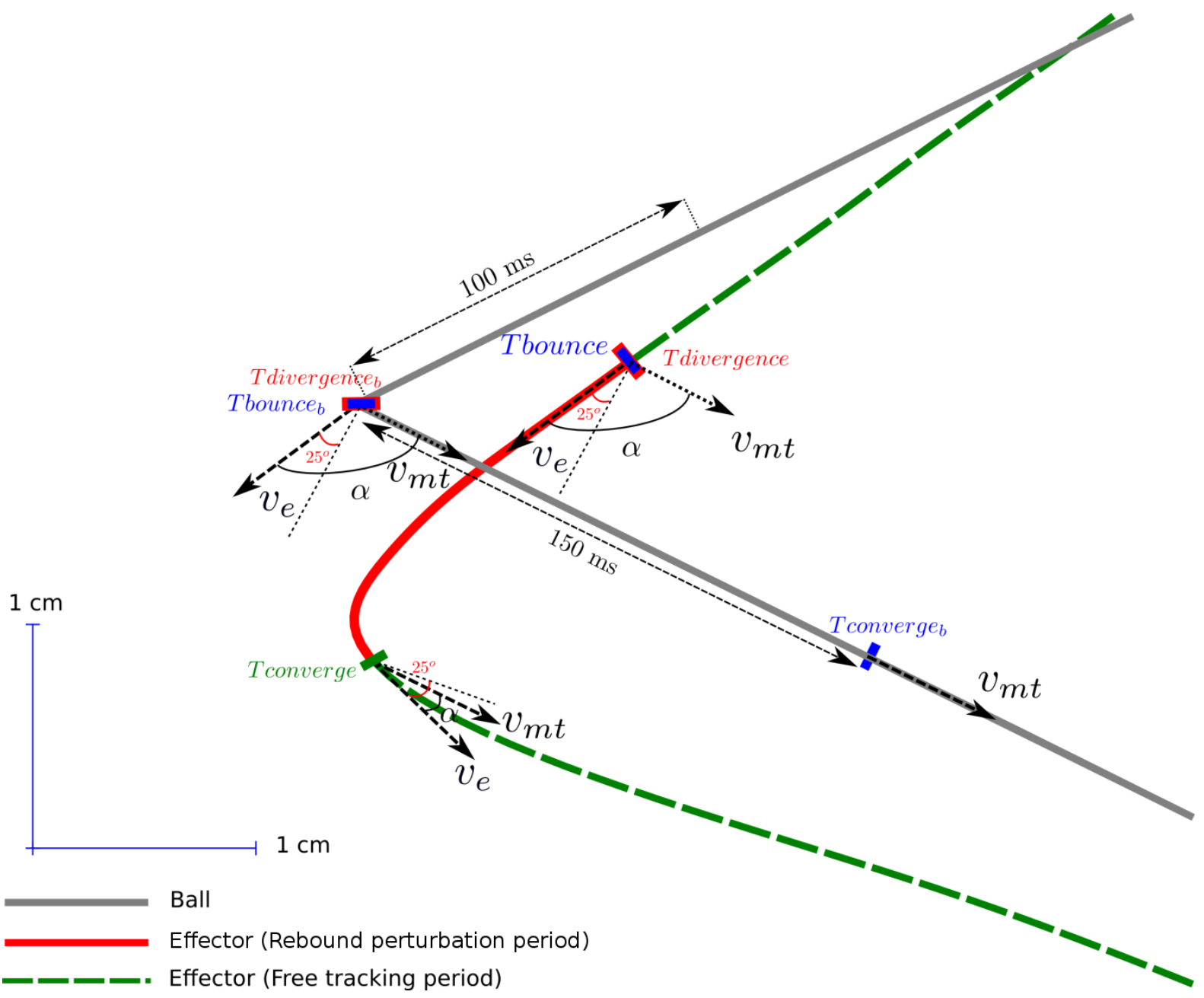

9

Figure 1 Illustration of various indicators to identify rebound perturbation period and free

11 tracking period. $\alpha=\arccos \left(\frac{V m t}{\|V m t\|}, \frac{V e}{\|V e\|}\right)$ with $\mathrm{V}_{\mathrm{mt}}$ and $\mathrm{V}_{\mathrm{e}}$ corresponding respectively to the

12 Vector moving Target and to the Vector Effector. The Time of divergence (Tdivergence) was 
1 determined when $\alpha>=25^{\circ}$. Whereas the time of convergence (Tconvergence) was determined

2 when $\alpha<25^{\circ}$. rebound perturbation $=$ [ Tdivergence - Tconvergence].

3

4

Distance to target was calculated to evaluate performance during tracking. This measure corresponded to the average distance (in $\mathrm{cm}$ ) between the effector and the moving target. Number of gap reductions referred to the number of times per second when participants reduced the distance between the effector and the target.

\section{Data Analysis}

All analyses were performed with the Matlab programming software based on an initial low-pass Butterworth filter with a cut off frequency of $10 \mathrm{~Hz}$. Initiation time, first interception time and duration of rebound perturbation period were analyzed using a mixed factorial analysis of variance (ANOVA) with Expertise as a between-subject effect (SuperExp vs. Exp vs. Non-Exp) x Acceleration (Constant velocity; Accelerated velocity) as a within-subject effect. Distance to target and number of gap reductions were analysed using an Expertise (Super-Exp vs. Exp vs. Non-Exp) $\times$ tracking period (rebound perturbation period; free tracking period). $\times$ Acceleration (Constant velocity; Accelerated velocity) analysis ANOVA with mixed-design. Differences in temporal and kinematic movement variables were statistically examined between the groups as a function of the different conditions (tracking period and acceleration conditions). Then, we evaluated the origin of the distance to target performance variable for each participant using a forward stepwise regression with initiation time, rebound perturbation period, number of gap reductions and time-to-peak-velocity as predictors. Statistical significance was set at $\mathrm{p}<.05$ for all tests. Newman-Keuls post hoc analyses were used when necessary to follow-up main and interaction effects.

\section{Results}

The initial temporal and kinematic characteristics of the effector are summarised in 
1 Table 1. Analysis of initiation time revealed a significant effect for Expertise $(F(2,39)=4.55$,

$\left.2 P<.05, \eta^{2}=.14\right)$. Post hoc analyses indicated a significant difference between Non-Exp (207

$3 \pm 23 \mathrm{~ms})$ and Exp (189 $\pm 15 \mathrm{~ms})$ as well as Super-Exp $(187 \pm 19 \mathrm{~ms})$, but no significant

4 difference between Exp and Super-Exp (Table 1). There was also a significant effect of 5 Expertise on first interception time $\left(\mathrm{F}(2,39)=3.71, P<.05, \eta^{2}=.16\right)$. Super-Exp had a 6 shorter first interception time than both Exp and Non-Exp $\left(\mathrm{M}_{\text {Super-Exp }}=482 \pm 98 \mathrm{~ms} ; \mathrm{M}_{\mathrm{Exp}}=\right.$ $7546 \pm 116 \mathrm{~ms} ; \mathrm{M}_{\text {Non-Exp }}=556 \pm 95 \mathrm{~ms}$ ). The results also revealed a significant effect for 8 acceleration $\left(\mathrm{F}(1,39)=21.21, P<.05, \eta^{2}=.35\right)$. Participants had a shorter first interception 9 time in the constant velocity conditions (490 $\pm 95 \mathrm{~ms})$ than accelerated conditions (570 \pm 105 ms). Movement time only showed a significant effect for acceleration $(\mathrm{F}(1,39)=28.13, P<$ $\left..05, \eta^{2}=.41\right)$. Participants had a shorter movement time in the constant velocity conditions $(292 \pm 80 \mathrm{~ms})$ than in the accelerated conditions (378 $\pm 102 \mathrm{~ms})$. Time-to-peak-velocity showed a significant effect for Expertise $\left(F(2,39)=11.09, P<.05, \eta^{2}=.36\right)$. Post hoc analyses showed a significant difference between three groups (Non-Exp (479 $\pm 46 \mathrm{~ms}$ ); Exp (440 $\pm 49 \mathrm{~ms})$; Super-Exp $(409 \pm 44 \mathrm{~ms})$ ). Peak velocity value only showed a significant effect for Acceleration $\left(\mathrm{F}(1,39)=7.56, P<.05, \eta^{2}=.16\right)$. Participants have a smaller peak velocity value in the constant velocity conditions $(7.53 \pm 1.02 \mathrm{~cm} / \mathrm{s})$ than accelerated conditions $(7.89 \pm 1.01 \mathrm{~cm} / \mathrm{s})$. 
1 Table 1: Characteristics of temporal and kinematic variables for the different groups of

4

5

6

7 expertise in the initiation of movement.

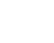

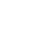

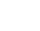

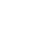

7

\begin{tabular}{l|c|c|c|} 
& Non-Exp & Exp & Super-Exp \\
\cline { 2 - 4 } Initiation time (ms) & $207 \pm 28$ & $189 \pm 17$ & $187 \pm 21$ \\
Movement Time (ms) & $349 \pm 87$ & $357 \pm 116$ & $295 \pm 91$ \\
First interception time (ms) & $556 \pm 95$ & $546 \pm 116$ & $482 \pm 98$ \\
Time-to-peak velocity (ms) & $479 \pm 46$ & $440 \pm 49$ & $409 \pm 44$ \\
Peak velocity value (cm/s) & $7.74 \pm 0.8$ & $7.40 \pm 0.67$ & $8.02 \pm 1.43$
\end{tabular}

Distance to target analysis revealed a significant effect of Expertise $(F(2,39)=6.66, P$ $\left.<.05, \eta^{2}=.25\right)$ : Non-Exp $(0.72 \pm 0.13 \mathrm{~cm})$ had a significantly greater distance to target than $\operatorname{Exp}(0.61 \pm 0.08 \mathrm{~cm})$ and Super-Exp $(0.57 \pm 0.11 \mathrm{~cm})$ but no significant difference was observed between Super-Exp and Exp. The results also revealed a significant effect of the tracking period $\left(\mathrm{F}(1,39)=39.59 P<.05, \eta^{2}=.50\right)$ and of Acceleration $(\mathrm{F}(1,39)=84.67, P<$ $\left..05, \eta^{2}=.68\right)$. Participants had a smaller Distance to target in the free tracking period than during the rebound perturbation period (respectively, 0.61 vs. $0.66 \mathrm{~cm}$ ) and for constant velocity than for accelerated velocity $(0.53 \pm 0.13$ vs. $0.74 \pm 0.16 \mathrm{~cm})$. The tracking period $\times$ Acceleration interaction revealed a significant effect $\left(F(1,39)=57.02, P<.05, \eta^{2}=.59\right)$. Post hoc analyses demonstrated that the difference between tracking period emerged only for the accelerated condition, participants had a longer distance to target in the rebound perturbation period $(0.79 \pm 0.17 \mathrm{~cm})$ than during the free tracking period $(0.68 \pm 0.14 \mathrm{~cm})$ (Figure 2). No other significant interactions were observed. 


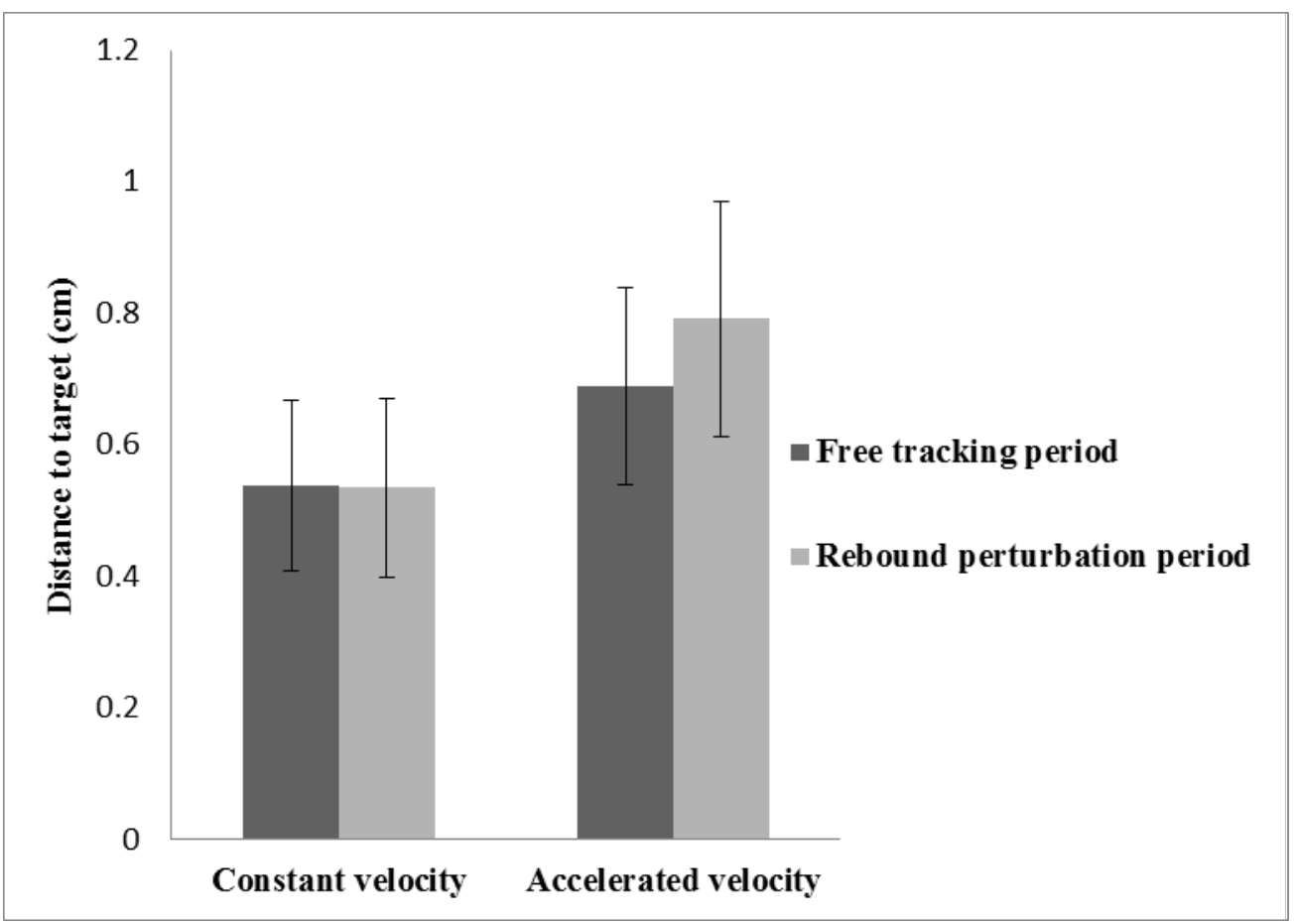

Figure 2 Distance to target according to tracking period and acceleration.

Rebound perturbation period analysis showed a significant effect of Expertise (F $\left.(2,39)=7.7, P<.05, \eta^{2}=.28\right)$. Post hoc analyses showed a significant difference between Non-Exp (228 $\pm 29 \mathrm{~ms})$ and $\operatorname{Exp}\left(\mathrm{M}_{\mathrm{Exp}}=203 \pm 19 \mathrm{~ms}\right)$ as well as Super-Exp (193 $\left.\pm 22 \mathrm{~ms}\right)$, but no significant difference between Exp and Super-Exp. There was also a significant effect for Acceleration $\left(\mathrm{F}(1,39)=10.58, \mathrm{P}<.05, \eta^{2}=.21\right)$ revealing that the rebound perturbation period was longer in the accelerated condition (217 $\pm 26 \mathrm{~ms}$ ) than in the constant velocity condition (201 $\pm 37 \mathrm{~ms})$. Number of gap reductions analysis showed a significant effect of Expertise $\left(F(2,39)=4.92, P<.05, \eta^{2}=.20\right)$. Post hoc testing showed a significant difference between Non-Exp $\left(\mathrm{M}_{\text {Non-Exp }}=2.57 \pm 0.63\right)$ vs. $\operatorname{Exp}\left(\mathrm{M}_{\operatorname{Exp}}=2.84 \pm 0.91\right)$ and Non-Exp vs. Super-Exp $\left(\mathrm{M}_{\text {Super-Exp }}=2.90 \pm 0.91\right)$, but no significant difference between Exp and SuperExp. A significant effect for the tracking period $\left(\mathrm{F}(1,39)=163.30, P<.05, \eta^{2}=.80\right)$ and of Acceleration $\left(\mathrm{F}(1,39)=7.21, P<.05, \eta^{2}=.15\right)$. Participants produced a higher number of gap reductions for the rebound perturbation period than free tracking period ( $3.47 \pm 0.78$ vs. 
$12.06 \pm 0.28)$ and for accelerated velocity in comparison with constant velocity (2.85 \pm 0.99 vs.

$22.68 \pm 0.83)$. The tracking period $\times$ Expertise interaction was significant $(\mathrm{F}(2,39)=3.68, P<$

$\left.3.05, \eta^{2}=.15\right)$. Post hoc revealed that the group difference occurred only in the rebound

4 perturbation period. Specifically, Non-Exp $(3.07 \pm 0.55)$ demonstrated a smaller number of

5 gap reductions in comparison with $\operatorname{Exp}(3.66 \pm 0.56)$ and Super-Exp (3.71 \pm 0.71$)$ (Figure 3).

$6 \quad$ No other interactions were observed.

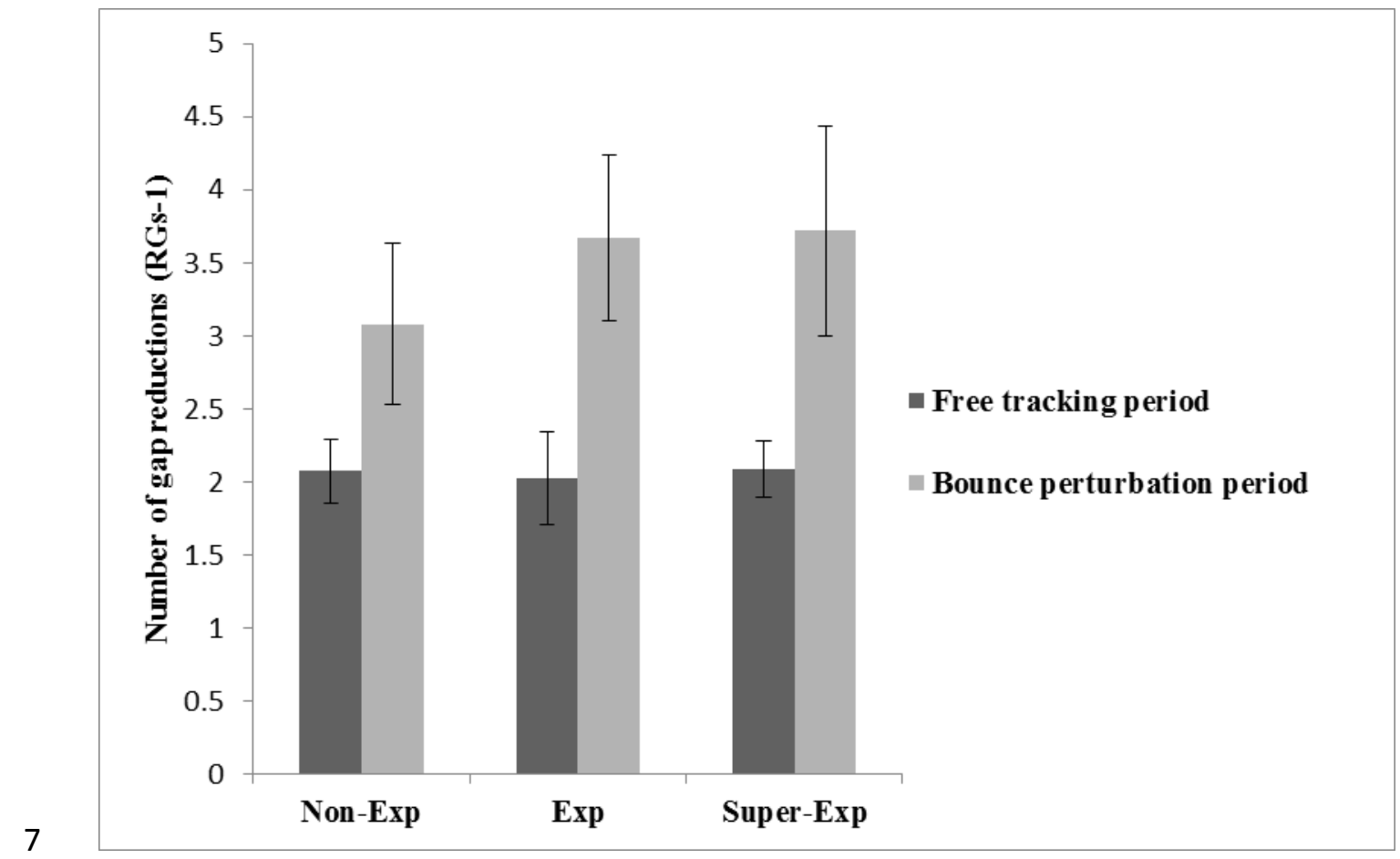

8 Figure 3 Number of gap reductions according to expertise and tracking period.

To determine whether the number of gap reductions could predict rebound perturbation period a simple regression was conducted and yielded the following relation: rebound perturbation period $=(-49.47 \mathrm{x}$ number of gap reductions $)+346.66, \mathrm{r}^{2}=.32$

\section{Predicting distance to target for Non-Exp, Exp and Super-Exp}

In order to determine which variables could explain the performance in the task (i.e.,

distance to target), we used a forward stepwise regression. For each participant, the distance

to target score was used as a dependent variable and initiation time, rebound perturbation 
1 period, number of gap reductions, and time-to-peak-velocity were used as independent

2 variables or predictors.

3 In the first step, the number of gap reductions was the best predictor of distance to target,

4 with a significant correlation .74 $(\mathrm{F}(1,40)=50.72)$, and explained 55\% of the total variance.

5 In the second step, rebound perturbation period was entered into the predictive equation and

6 was found to explain an additional $14 \%$ of the total variance. In the third step, initiation time

7 was added and explains $1 \%$ of the total variance. In final step, time-to-peak-velocity was

8 added and explains a supplementary 3\% of the total variance. In the final equation, number of

9 gap reductions $(\beta=-0.50)$, duration of rebound perturbation period $(\beta=0.44)$, initiation time

10 ( $\beta=0.23)$, and time-to-peak-velocity $(\beta=-0.22)$ explained $74 \%$ of the total variance, with

11 significant correlation of .86 $(\mathrm{F}(4,37)=25.90)$ (Figure 4$)$. 


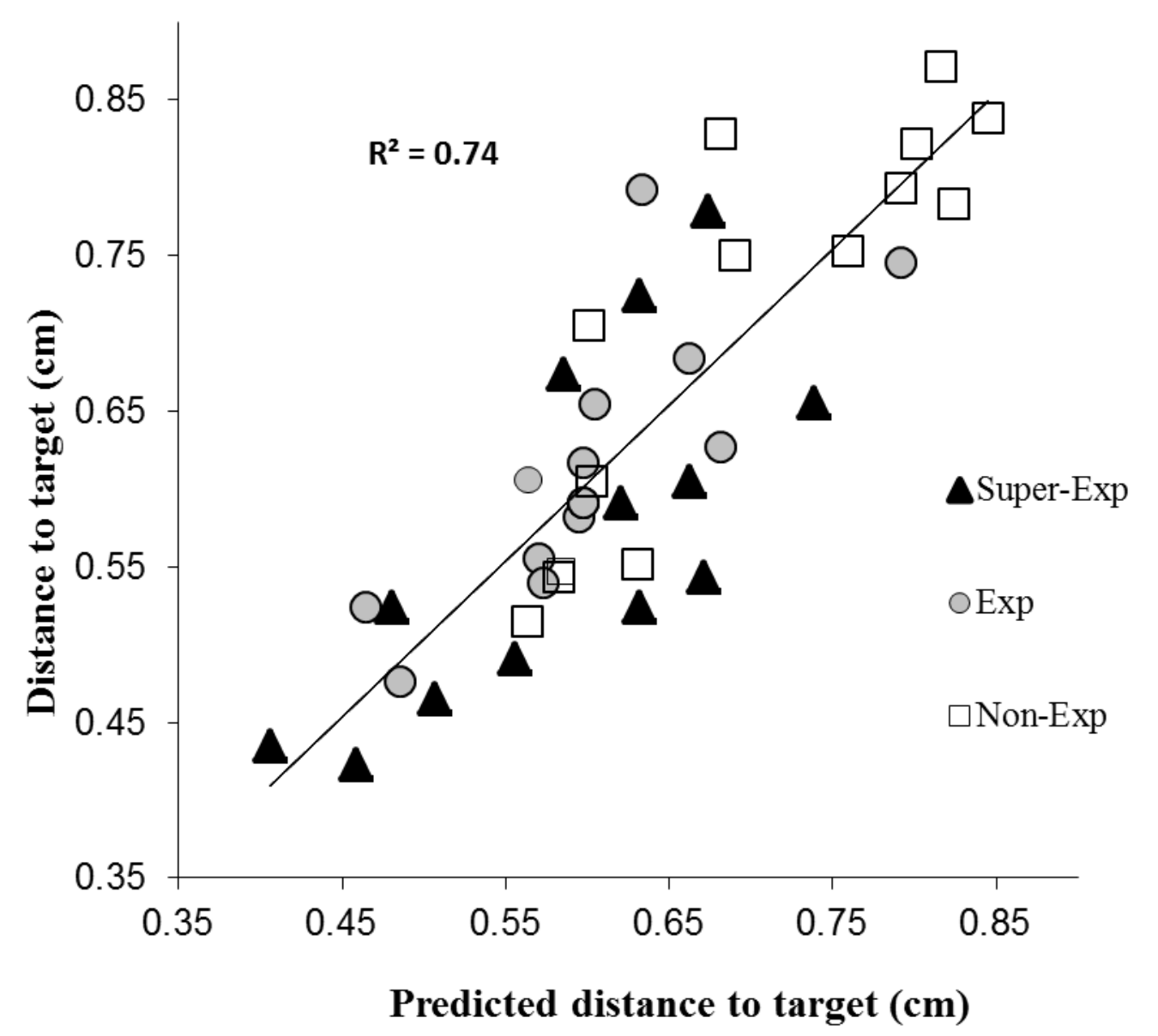

2 Figure 4 Distance to target as a function of predicted distance to target on the basis of

3 multiple regression analysis with duration of number of gap reductions, rebound perturbation

4 period, initiation time, and time-to-peak-velocity as predictors. The equation of this prediction

5 can be written as follows: Predicted distance to target $=[-0.19 \times$ number of gap reductions $]+$

$6[0.002 \mathrm{x}$ rebound perturbation period $]+[1.39 \mathrm{x}$ initiation time $]+[-0.57 \mathrm{x}$ time-to-peak-

7 velocity $]+[0.74]$.

8 Discussion

9 The purpose of this study was to examine whether perception-action coupling capacities are a possible determinant of expertise in fast-ball sports. We aimed to ascertain

11 whether these supposed superior capacities could be revealed through the completion of a VMT task that had a varying level of difficulty according to the rebounds of the target and the 
1

2

variation of the target velocity (e.g., Benguigui et al., 2013, Le Runigo et al., 2005; 2010). In addition to differences between Exp and Non-Exp ${ }^{1}$, we also aimed to examine whether differences between Exp and Super-Exp were revealed within the experiment.

The results revealed a significant effect of expertise on initiation time, first interception time and time to peak velocity. Super-Exp initiated their movement response and intercepted the target earlier than Exp and Non-Exp; likewise their peak velocity occurred earlier than Non-Exp. Movement time and peak velocity value did not reveal differences between the different groups of expertise. These results show that the shorter time to intercept the target is the result of a shorter latency in the adaptation of movement, which is in line with previous expertise studies in ball sports (e.g., McRobert, \& Tayler, 2005; Renshaw \& Fairweather, 2000). Analysis of distance to target showed that Experts performed better in the tracking task than Non-Exp. Even though the VMT task does not have the same demands of performance as on a tennis court, the results indicate that this task allows the discrimination of expertise and requires processes that may be commensurate with those required within fastball sports. The evidence from the current study indicates that it may not be necessary to sample sport specific situations within an experiment to reveal differences between experts and non-experts (see also, Faubert, 2013). That is, assuming that demanding laboratory tasks for perception-action coupling can be sufficient.

For all participants, the accuracy of pursuit decreased in the accelerated conditions. This observation is consistent with the hypothesis that the perceptual-motor system has greater difficulties in adapting to accelerated moving objects than those with constant velocities (e.g., Bennett \& Benguigui, 2013; 2016; Watamaniuk \& Heinen, 2003). It is known that interceptive actions are based on first-order information corresponding to the velocity

\footnotetext{
${ }^{1}$ As we had two groups of experts (Super-Exp and Exp), we use the term "Expert "in a general sense and SuperExp and Exp to discuss differences between the two groups.
} 
rather than second-order information corresponding to acceleration (e.g., Bootsma et al, 1997). Consequently, accelerated conditions require continuous adaptations of movement and require a stronger involvement of regulatory mechanisms (e.g., Benguigui et al., 2003). Although the occurrence of rebounds could be anticipated (i.e., participants could see that the target would contact the side of the display), they led to decreases in tracking accuracy which increased the distance to target during the rebound perturbation period. The experts outperformed non-experts in this condition with a smaller distance to target and a shorter duration of this period. This finding is in line with previous results, which have revealed that changes in the direction of a target increases difficulty in visuomotor coordination as they require a large degree of movement adaptation (Le Runigo et al., 2005, 2010). This highlights that deviations in ball trajectories, as a consequence of a rebounds, may be a particularly interesting situation with which to examine expertise in future work. Previously, eye-tracking studies have revealed that experts make specific fixations during bounce periods to pick-up information about the new direction of the ball leading to accurate interception (Ripoll \& Fleurance, 1988; Land \& McLeod, 2000).

The analysis of the number of gap reductions revealed greater movement regulation in Super-Exp compared to Non-Exp specifically during the tracking period, immediately before/after the rebound. The results lend support for the possibility that prospective regulation is a key determinant in the task. The larger number of gap reductions enhanced tracking accuracy and enabled the experts to be more precise in controlling their movements, as regulated by online information. The correlation between the number of gap reductions and the duration of the rebound perturbation period can be interpreted as evidence supporting this assumption. Confirming previous work (Bootsma \& van Wieringen, 1990; McLeod, 1987), these results suggest that Experts have optimised their perception-action coupling to regulate and adapt their movements in a more accurate manner. 
2 gap reductions variables were the best predictors of performance in the task. This analysis and the regression, as shown in Figure 4, offers a further means to understand expertise effects in the VMT task. Although ANOVA did not reveal significant differences in performances between Exp and Super-Exp groups, Figure 4 indicates that the three groups are well defined, with most of the Super-Exp placed on the bottom-left of the plot (which indicates better performance), most of Experts in the middle and Non-Exp on the upper-right of the plot (which indicates poorer performance). One can notice the presence of some variance with some Super-Exp and Non-Exp who had intermediate performances, while Exp were distributed between the Super-Exp and Non-Exp group performances (Figure 4). This stepwise regression analysis reveals the lack of homogeneity in the different groups, which might be explained by the multi-factorial characteristic of expertise and inter-individual variability between experts (Baker \& Davids, 2007). For instance, evidence indicates that expertise in sports is predicated on a number of interacting attributes including action capabilities (e.g., Dicks et al., 2010b), psychological skills (e.g., Thelwell et al., 2007) or physiological characteristics (e.g., Joyner \& Coyle, 2008). Thus, experts could have a relative weakness in their visuomotor coordination, and subsequently they could compensate for this through strengths in other attributes. Consequently, variations in such characteristics are likely to give rise to differences in perceptual capacities (Withagen \& Chemero, 2009).

The VMT task used in the current experiment was a simulated interceptive task, which prevents specific generalizations being made about performance in sport specific contexts. However, the functional coupling between perception and action demanded by the task was sufficient to reveal an expertise effect. For instance, the bounce tracking period simulated trajectory deviations that may be comparable to those experienced in sport situations (e.g., bounce in tennis game). Future work could focus on testing the paradigm of deviated 
1

trajectories with not only expected but also unexpected deviations and to examine perceptualmotor skills in a high-dimensional context such as in a 3D virtual reality experiment (Bideau et al., 2010). Such constraints could allow the discrimination of different level of expertise which could provide an opportunity to develop tests of talent identification. This also opens the question about the possibility of developing virtual reality tasks to train perceptual-motor skills (Faubert, 2013). Although it is likely that such technologies would only offer a supplement to typical training methods, there are suggestions that such training may be beneficial to athletes, particularly as part of rehabilitation programs (Appelbaum \& Erickson, 2016).

To conclude, the results from the current study suggest that the VMT task allowed the discrimination of tennis expertise. Experts initiated earlier movements to adapt and to regulate their actions in comparison with Non-Exp and therefore, they appeared to be able to transfer their perception-action coupling capacities to the VMT task. These results are in accordance firstly with the temporal hypothesis that referred to earlier movement initiation and less delay in movement adaptations for experts and secondly with the movement kinematic adaptation hypothesis, that referred to a better ability for experts to regulate actions (Le Runigo et al., 2005, 2010). The differences within experts were highlighted in the most complex and demanding conditions of movement control confirming that a part of expertise lies in the ability to develop prospective control. 


\section{References}

Appelbaum, L. G., \& Erickson, G. (2016). Sports vision training: A review of the state-of-the-art in digital training techniques. International Review of Sport and Exercise Psychology, 1-30.

Bavelier, D. (2006). Video Games as a Tool to Train Cognitive Skills. ROCHESTER UNIV NY.

Le Runigo, C., Benguigui, N., \& Bardy, B. G. (2010). Visuo-motor delay, information-movement coupling, and expertise in ball sports. Journal of sports sciences, 28(3), 327-337.

Benguigui, N., Ripoll, H., \& Broderick, M. P. (2003). Time-to-contact estimation of accelerated stimuli is based on first-order information. Journal of Experimental Psychology: Human Perception and Performance, 29(6), 1083.

Bennett, S. J., \& Benguigui, N. (2013). Is acceleration used for ocular pursuit and spatial estimation during prediction motion?. PloS one, 8(5), e63382.

Bennett, S. J., \& Benguigui, N. (2016). Spatial Estimation of Accelerated Stimuli Is Based on a Linear Extrapolation of First-Order Information. Experimental psychology.

Bideau, B., Kulpa, R., Vignais, N., Brault, S., Multon, F., \& Craig, C. (2010). Using virtual reality to analyze sports performance. IEEE Computer Graphics and Applications, 30(2), 14-21.

Bootsma, R. J., Fayt, V., Zaal, F. T., \& Laurent, M. (1997). On the information-based regulation of movement: What Wann (1996) may want to consider. Journal of Experimental Psychology: Human Perception and Performance, 23(4), 1282.

Bootsma, R. J., \& Van Wieringen, P. C. (1990). Timing an attacking forehand drive in table tennis. Journal of experimental psychology: Human perception and performance, 16(1), 21. 
Davids, K., \& Baker, J. (2007). Genes, environment and sport performance. Sports Medicine, 37(11), 961-980.

Dicks, M., Davids, K., \& Button, C. (2010). Individual differences in the visual control of intercepting a penalty kick in association football. Human Movement Science, 29(3), 401-411.

Dicks, M., Davids, K., \& Button, C. (2010). Individual differences in the visual control of intercepting a penalty kick in association football. Human Movement Science, 29(3), 401-411.

Ericsson, K. A., \& Smith, J. (1991). Toward a general theory of expertise: Prospects and limits. Cambridge University Press.

Faubert, J. (2013). Professional athletes have extraordinary skills for rapidly learning complex and neutral dynamic visual scenes. Scientific reports, 3, 1154.

Gray, R. (2002). Behavior of college baseball players in a virtual batting task. Journal of Experimental Psychology: Human Perception and Performance, 28(5), 1131.

Green, C. S., \& Bavelier, D. (2006). Enumeration versus multiple object tracking: The case of action video game players. Cognition, 101(1), 217-245.

Jackson, R. C., \& Mogan, P. (2007). Advance visual information, awareness, and anticipation skill. Journal of motor behavior, 39(5), 341-351.

Jacobs, D. M., \& Michaels, C. F. (2006). Lateral interception I: operative optical variables, attunement, and calibration. Journal of Experimental Psychology: Human Perception and Performance, 32(2), 443.

Joyner, M. J., \& Coyle, E. F. (2008). Endurance exercise performance: the physiology of champions. The Journal of physiology, 586(1), 35-44.

Le Runigo, C., Benguigui, N., \& Bardy, B. G. (2005). Perception-action coupling and expertise in interceptive actions. Human Movement Science, 24(3), 429-445. 
2 information-movement coupling, and expertise in ball sports. Journal of sports sciences,

3

4

5 28(3), 327-337.

McLeod, P. (1987). Visual reaction time and high-speed ball games. Perception, 16(1), 49-59.

McRobert, A., \& Tayler, M. (2005). Perceptual abilities of experienced and inexperienced cricket batsmen in differentiating between left hand and right hand bowling deliveries. Journal of Sports Sciences, 23(2), 190-191.

Michaels, C. F., Jacobs, D. M., \& Bongers, R. M. (2006). Lateral interception II:

Predicting hand movements. Journal of Experimental Psychology-Human Perception and Performance, 32(2), 459-472.

Renshaw, I., \& Fairweather, M. M. (2000). Cricket bowling deliveries and the discrimination ability of professional and amateur batters. Journal of Sports Sciences, 18(12), 951-957.

Thelwell, R. C., Weston, N. J., \& Greenlees, I. A. (2007). Batting on a sticky wicket: Identifying sources of stress and associated coping strategies for professional cricket batsmen. Psychology of Sport and Exercise, 8(2), 219-232.

Triolet, C., Benguigui, N., Le Runigo, C., \& Williams, A. M. (2013). Quantifying the nature of anticipation in professional tennis. Journal of Sports Sciences, 31(8), 820-830. Watamaniuk, S. N., \& Heinen, S. J. (2003). Perceptual and oculomotor evidence of limitations on processing accelerating motion. Journal of vision, 3(11), 5-5.

Chase, W. G., \& Simon, H. A. (1973). Perception in chess. Cognitive psychology, $4(1), 55-81$. 
Williams, A. M., \& Ericsson, K. A. (2005). Perceptual-cognitive expertise in sport:

2 Some considerations when applying the expert performance approach. Human movement 3 science, 24(3), 283-307.

4

Williams, A. M., Ward, P., Knowles, J. M., \& Smeeton, N. J. (2002). Anticipation

5 skill in a real-world task: measurement, training, and transfer in tennis. Journal of

$6 \quad$ Experimental Psychology: Applied, 8(4), 259.

7

Withagen, R., \& Chemero, A. (2009). Naturalizing perception: Developing the

8 Gibsonian approach to perception along evolutionary lines. Theory \& Psychology, 19(3), 363-

9389.

Yarrow, K., Brown, P., \& Krakauer, J. W. (2009). Inside the brain of an elite athlete:

11 the neural processes that support high achievement in sports. Nature Reviews Neuroscience, 12 10(8), 585-596. 